\title{
Multiplexed fiber-optic Fabry-Pérot cavities for refractive index and temperature sensing fabricated using diamond-blade dicing
}

\author{
Ivonne Pfalzgraf ${ }^{1}$, Sergiy Suntsov ${ }^{1}$, Kore Hasse ${ }^{1}$, and Detlef Kip $^{1, *}$ \\ ${ }^{1}$ Faculty of Electrical Engineering, Helmut Schmidt University, 22043 Hamburg, Germany
}

\begin{abstract}
We report on multiplexing several Fabry-Pérot (FP) cavities in single-mode optical fibers for highprecision spatially resolved sensing of refractive indices (RI) of liquids. Resonators are fabricated by cutting small slots into fibers using a diamond-blade dicing saw and additional coating with thin $\mathrm{Ta}_{2} \mathrm{O}_{5}$ layers to increase cavity reflectance. Temperature compensation of RI measurements is achieved either by evaluating the reflection signals resulting from the solid core parts between different open-cavity sensor elements, or by using a thin Si inlay glued into one of the open cavities. The multiplexing performance and accuracy of the fabricated sensors with up to four open cavities were tested on sucrose solutions over a range of temperatures.
\end{abstract}

\section{Introduction}

Fiber-optic sensors have gained much attention in diverse areas due to their excellent characteristics including small size, high sensitivity, biocompatibility, and immunity to electromagnetic interference [1-4]. Among them, fiber sensors based on FP interference have shown very high sensitivity for measuring different physical parameters like RI, pressure, or temperature. At the same time, there is an interesting option for multiplexing of several sensors in a single fiber. Cutting open cavities into an optical fiber and through its core is perhaps the easiest realization of FP resonators. Here blade diced cavities [4-6] constitutes a simple alternative to other methods, such as chemical etching, laser micromachining, ion beam milling, or fiber splicing.

A possible application of multiplexed FP sensors at the fiber tip is the detection of the spatial distribution inside a liquid under test. Furthermore, FP cavities can be filled with molecularly imprinted polymers or coated with functional layers sensitive to trace substances, allowing the realization of an ultra-compact lab-on-a-fiber.

In this work, we present multiplexing of four opencavity FP RI sensors in one optical fiber, clearly exceeding our recent results described in [6]. Individual sensors are formed by dicing through the core of a standard step-index single-mode fiber using a precision diamond-blade wafer saw. Either the solid fiber parts between open cavities or an additional Si inlay glued into one of the cavities can be used for temperature sensing [2]. To test the sensor's performance and the ability to sense refractive index of solutions with high precision at different spatial locations, we perform temperature dependent measurements in sucrose solutions with precisely adjusted refractive index.

\section{Fabrication Method and Setup}

For cutting the optical fiber is glued into a quartz ferrule to increase mechanical stability. Slots of different width are cut through the core of the optical fibers using a Disco DAD322 wafer saw to realize open FP micro-cavities. To achieve smooth surfaces, resin-based blades with very fine grit are used. In order to increase the mechanical stability of the whole sensor structure with its many cuts, a surrounding quartz glass ferrule is used. Due to blade conditioning with a special dresser board, all cuts have a nearly rectangular shape. For an optional $\mathrm{Si}$ inlay a rectangular plate is prepared with the dicing saw, placed inside one of the open cavities and glued with NOA61. The reflectivity of open FP cavities can be increased using dielectric layers on both sidewalls. For this we evaporated single high-index $\mathrm{Ta}_{2} \mathrm{O}_{5}$ layers $(n=2.07$ at $1550 \mathrm{~nm})$ on

a)
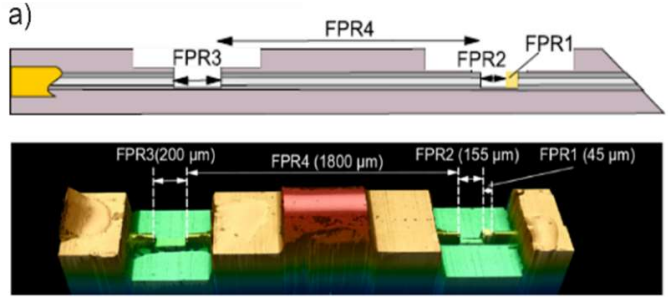

b)

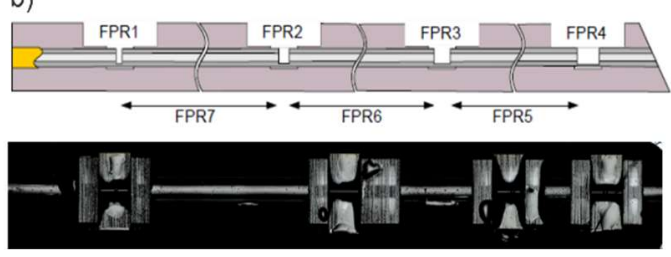

Fig. 1. Examples of two FP fiber sensors (top: schematic; bottom: confocal microscope image): (a) two RI sensors with additional Si inlay, (b) four different open-cavity RI sensors.

\footnotetext{
* Corresponding author: kip@hsu-hh.de
} 
both facets of an open cavity while monitoring the optical reflection spectra inside an electron-beam coater. In Fig. 1 two examples of fabricated sensors are shown.

For the experimental setup an interrogation system (Micron Optics Hyperion Si155) operating in the wavelength range of $(1460-1620) \mathrm{nm}$ with an accuracy of $1 \mathrm{pm}$ and a computer system for data read-out is used. The refractive index of the medium under test is changed by using sucrose solutions with calibrated RI in a temperature stabilized water bath (Julabo FP45) having an accuracy of $0.01^{\circ} \mathrm{C}$.

In order to extract small changes of the optical length $L_{o}$ of the individual cavities induced by temperature $T$ and/or RI changes, phase tracking of the essential fast Fourier transform (FFT) components is used. Temperature and RI changes are calculated from the measured total phase changes $\Delta \Phi$ using appropriate calibration functions:

$$
\Delta n=\frac{\lambda}{4 \pi L_{R}} \Delta \phi=\Delta n_{F}(T)+\Delta n_{L}(T)=\Delta n_{m}+f_{1}
$$

where $\Delta n_{m}$ is the RI change related to the composition of the water based liquid (e.g. the concentration of sucrose in water), $\Delta n_{F}$ is the temperature dependent fluid's RI change and $\Delta n_{L}$ is a contribution caused by the sensor's thermal expansion $\Delta L_{R}$ of the physical cavity length $L_{R}$. The two temperature dependent parts can be combined in a temperature calibration function $f_{1}$.

Calibration of the temperature-sensing solid core regions is performed using

$$
\Delta T_{m}=\frac{\lambda}{4 \pi(d n / d T) L_{R}} \Delta \phi f_{2}=\Delta T f_{2}
$$

where $\Delta T_{m}$ is the temperature measured by the fiber parts, $d n / d T$ is the thermo-optic coefficient of silica, and $f_{2}$ is another calibration function.

\section{Experimental Results}

The sensing region of the first sensor from Fig. 1 comprises four adjacent FPRs, namely the two open cavities, the solid fiber part in between and the Si inlay. To determine the sensitivity of the open cavities, the sensor was first immersed in solutions with different RI. Figure 2 (a) shows the measured phase change and calculated change in RI for the two open cavities, resulting in a sensitivity of $1153 \mathrm{~nm} / \mathrm{RIU}$ for FPR2 and $1168 \mathrm{~nm} / \mathrm{RIU}$ for FPR3. Here the sensor is calibrated in order to measure value $\Delta \mathrm{n}_{\mathrm{m}}$ without cross-sensitivity to temperature. The response of the $\mathrm{Si}$ inlay and of the fiber piece between FPR2 and FPR3 to temperature changes is presented in Fig. 2 (b). The temperature is measured with a spectral sensitivity of $79 \mathrm{pm} /{ }^{\circ} \mathrm{C}$ for the $\mathrm{Si}$ inlay and a lower value of $13 \mathrm{pm} /{ }^{\circ} \mathrm{C}$ for the fiber piece. Here the high sensitivity of the Si inlay results from the large thermooptic coefficient $1.7 \times 10^{-4} \mathrm{~K}^{-1}$ of Si. Overall, this sensor allows for spatially resolved temperature-compensated RI sensing with an accuracy of $2 \times 10^{-5}$ RIU.
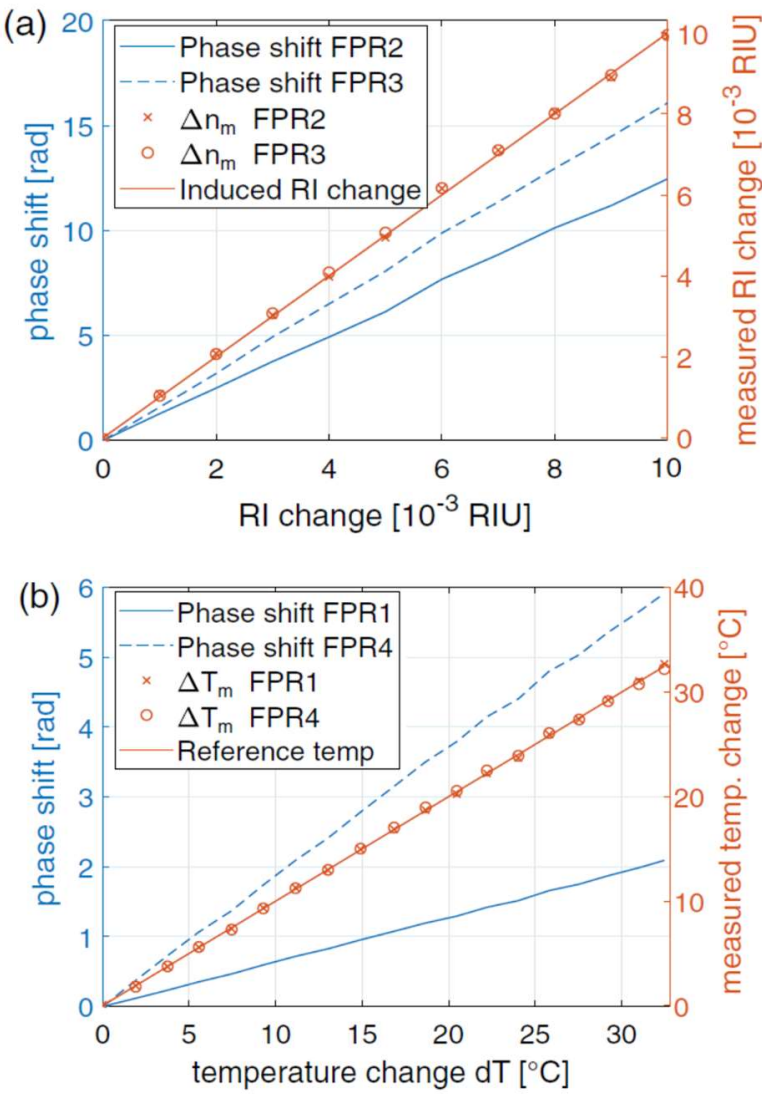

Fig. 2. (a) Response of the open cavities to a change in RI, induced with sucrose solutions. (b) Response of Si inlay and fiber piece for an induced change in temperature.

In the second example, we present a fiber sensor where we explored a higher number of accurately evaluable open cavities on a single fiber with additional enhancement of their reflectivity using dielectric coatings. The sensor is measuring simultaneously refractive index distributions using four different open cavities distributed along the fiber. As before, additional FP resonators formed by solid fiber parts in between the open cavities are used for temperature measurement. After appropriate calibration, this allows for automatic compensation of crosstalk between temperature- and composition-induced fluids' refractive index changes. Starting with FP resonator FPR4 ( $1^{\text {st }}$ coating) located towards the tilted tip of the fiber (used to avoid reflections) and afterwards FPR3 $\left(2^{\text {nd }}\right.$ coating) were additionally coated with thin $\mathrm{Ta}_{2} \mathrm{O}_{5}$ layers, while the reflection spectra of the sensor were simultaneously recorded during evaporation. While the optical thickness of the coating for sensor FPR4 was chosen to match a quarter-wave layer. The thicknesses for the coatings of FPR3 were chosen to be significantly lower and are about $1 / 6.5$ of the thickness of that for FPR4. In this way, the response (i.e. strength of corresponding FFT peak) of each open cavity was optimized in order to improve the accuracy of RI measurements for both FPR3 and FPR4. The obtained experimental FFT spectra of Fig. 3 are in good quantitative agreement with the simulated ones. The slight increase in the reflectivity of FPR2 is here probably due to insufficient shielding of this sensor in the second coating step. 


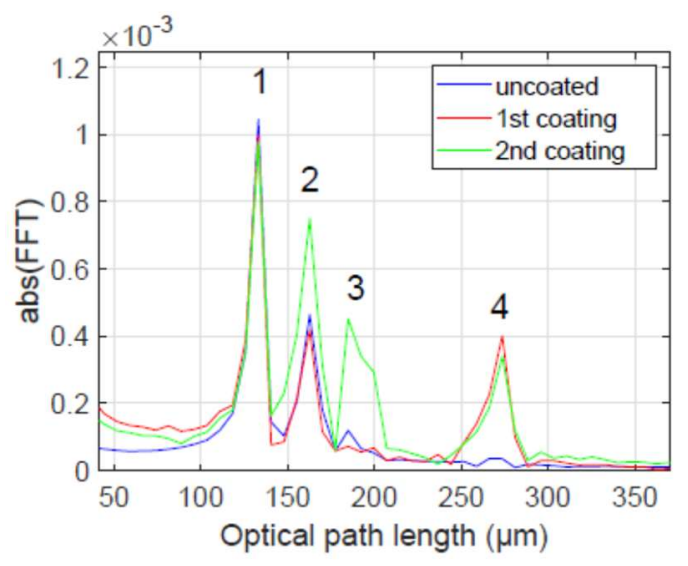

Fig. 3. Calculated FFT of the measured reflection spectra of the sensor with different configurations of coatings while immersed in DI water: no coating (blue), coating on the side walls of FPR4 (red) and additional FPR3 (green).

The RI sensing properties of this multiplexed sensor are presented in Fig. 4. Obviously, the accuracy of RI measurements is significantly enhanced by additional reflective coatings (cf. Fig. 4(b)) when compared to the initially uncoated ones (cf. Fig. 4(a)). The achieved sensitivity of $\sim 1185 \mathrm{~nm} / \mathrm{RIU}$ for RI sensing is similar for all four sensors. Again, calibration of the sensor allows for full compensation of any temperature crosssensitivity.

(a)

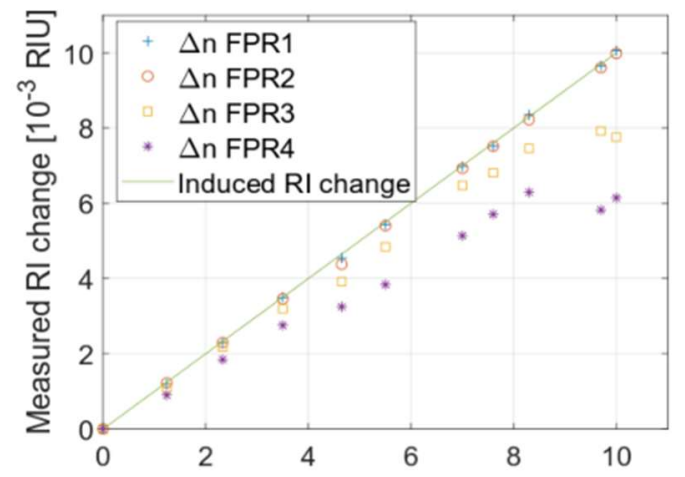

(b)

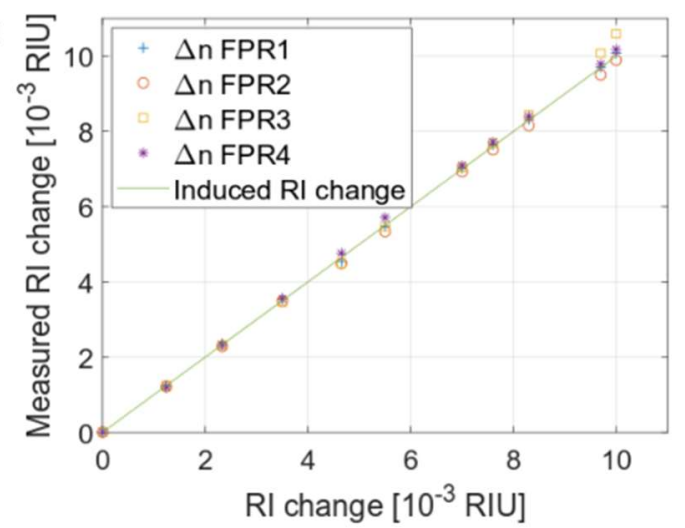

Fig. 4. Measurement of RI of the same liquid using the sensor from Fig. 1 (b) with four independent FP sensors with (a) uncoated FPRs) and (b) coated sensors FPR3 and FPR4.

Next, we characterize this sensor in terms of its temperature measurement sensitivity using the solid core fiber sections. For this, in Fig. 5 we determined the response (phase change) to induced temperature $\left(0.5^{\circ} \mathrm{C}\right.$ steps) changes when the senor is immersed in DI water. Sensitivities of $19.4 \mathrm{pm} /{ }^{\circ} \mathrm{C}(\mathrm{FPR} 5), 19.33 \mathrm{pm} /{ }^{\circ} \mathrm{C}$ (FPR6) and $20 \mathrm{pm} /{ }^{\circ} \mathrm{C}$ (FPR7) were achieved with an accuracy of $0.01 /{ }^{\circ} \mathrm{C}$. This sensor thus shows high average sensitivities of $1160 \mathrm{~nm} / \mathrm{RIU}$ and $20 \mathrm{pm} /{ }^{\circ} \mathrm{C}$ for RI and temperature, respectively, with good suppression of cross-talk among measured parameters and sensor elements, as required for biomedical applications.

Finally, with the performed calibration of the different sensing regions for either RI or temperature changes, we test the cross-sensitivity of the open cavities FPR1 to

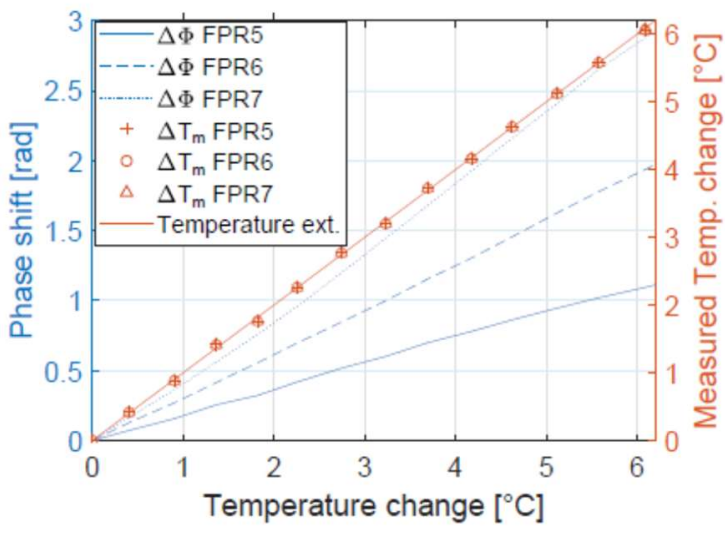

Fig. 5. Response of the solid fiber sections for an induced change in temperature.

FPR4 with respect to temperature. For this, the sensor is place in DI water while the temperature is changed. From this measurement shown in Fig. 6, we determine the accuracy, i.e. the deviation of the values $\Delta n_{m}$ measured in this way from the expected value $\Delta n_{m}=0$ is in the range of $6 \times 10^{-6}$ RIU.

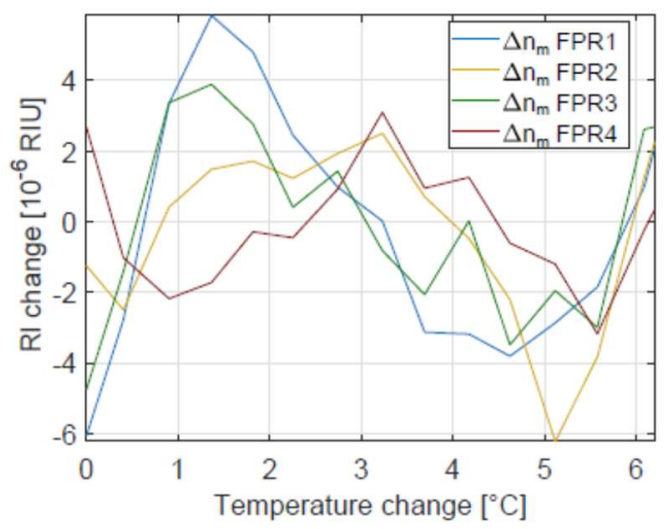

Fig. 6. Measurement of corrected RI change $\Delta n m$ after temperature calibration of all open-cavity sensors.

\section{Conclusion}

In summary, we have demonstrated a very compact sensor at the tip of a single-mode optical fiber, with which four open FP cavities can be read out simultaneously with high accuracy. At the same time, real-time temperature compensation is possible, thus avoiding any cross-talk induced by inherent temperature changes during a measurement. 
Such multiplexed fiber sensors can be effectively fabricated by diamond-blade dicing into single-mode fibers and have great potential to be used as lab-on-afiber. Additional reflective coatings allow for an improved accuracy, while the solid fiber parts can be applied to compensate for any cross-talk by temperature changes. Due to its compact dimensions, this novel fiber sensor may suit ideally to monitor both RI and temperature changes in small volume samples like in biomedical applications.

Funding by Deutsche Forschungsgemeinschaft (DFG) (KI482/19-1) is gratefully acknowledged.

\section{References}

1. H. Joe, H. Yun, S. Jo, B. Min, Mater. Struct. 5, 173 (2018)

2. B. Lee, Opt. Fiber Technol. 9, 57 (2003)

3. S. Suntsov, C. E. Rüter, D. Kip, Appl. Opt. 58, 2076 (2019)

4. H. Bae, D. Yun, H. Liu, D. A. Olson, M. Yu, J. Lightwave Technol. 32, 1585 (2014)

5. S. Suntsov, C. E. Rüter, T. Schipkowski, D. Kip, Appl. Opt. 56, 9139 (2017)

6. I. Pfalzgraf, S. Suntsov, D. Kip, Appl. Opt. 60, 1428 (2021) 